\title{
Hyperfine anomaly in gold and magnetic moments of $I^{\pi}=11 / 2^{-}$gold isomers
}

A. E. Barzakh $\odot,,^{1, *}$ D. Atanasov,$^{2, \dagger}$ A. N. Andreyev, ${ }^{3,4}$ M. Al Monthery, ${ }^{3}$ N. A. Althubiti, ${ }^{5,6}$ B. Andel, ${ }^{7,8}$ S. Antalic, ${ }^{8}$ K. Blaum, ${ }^{2}$ T. E. Cocolios, ${ }^{7,5}$ J. G. Cubiss, ${ }^{3}$ P. Van Duppen, ${ }^{7}$ T. Day Goodacre,,${ }^{9,5}$, A. de Roubin,,${ }^{2,}$ Yu. A. Demidov, ${ }^{1,10}$ G. J. Farooq-Smith, ${ }^{7,5}$ D. V. Fedorov,${ }^{1}$ V. N. Fedosseev, ${ }^{9}$ D. A. Fink, ${ }^{9,2}$ L. P. Gaffney, ${ }^{9,11}$ L. Ghys, $,{ }^{7}, 12$ R. D. Harding, ${ }^{3,9}$ D. T. Joss, ${ }^{11}$ F. Herfurth, ${ }^{13}$ M. Huyse,${ }^{7}$ N. Imai, ${ }^{14}$ M. G. Kozlov, ${ }^{1,10}$ S. Kreim, ${ }^{9,2}$ D. Lunney, ${ }^{15, \|}$ K. M. Lynch, ${ }^{9,5}$ V. Manea, ${ }^{2, \|}$ B. A. Marsh, ${ }^{9}$ Y. Martinez Palenzuela, ${ }^{9,7}$ P. L. Molkanov, ${ }^{1}$ D. Neidherr, ${ }^{13}$ R. D. Page, ${ }^{11}$ M. Rosenbusch, ${ }^{16}$ R. E. Rossel,${ }^{9,17}$ S. Rothe, ${ }^{9,17}$ L. Schweikhard ${ }^{16}$ M. D. Seliverstov, ${ }^{1}$ S. Sels, ${ }^{7}$ C. Van Beveren, ${ }^{7}$ E. Verstraelen, ${ }^{7}$ A. Welker, ${ }^{9,18}$ F. Wienholtz, ${ }^{9,16}$ R. N. Wolf, ${ }^{2,16,4 l}$ and K. Zuber ${ }^{18}$

${ }^{1}$ Petersburg Nuclear Physics Institute, NRC Kurchatov Institute, 188300 Gatchina, Russia

${ }^{2}$ Max-Planck-Institut für Kernphysik, Saupfercheckweg 1, 69117 Heidelberg, Germany

${ }^{3}$ Department of Physics, University of York, York, YO10 5DD, United Kingdom

${ }^{4}$ Advanced Science Research Center (ASRC), Japan Atomic Energy Agency, Tokai-mura, Japan

${ }^{5}$ The University of Manchester, School of Physics and Astronomy, Oxford Road, M13 9PL Manchester, United Kingdom

${ }^{6}$ Physics Department, Faculty of Science, Jouf University, Aljouf, Saudi Arabia

${ }^{7}$ KU Leuven, Instituut voor Kern- en Stralingsfysica, 3001 Leuven, Belgium

${ }^{8}$ Department of Nuclear Physics and Biophysics, Comenius University in Bratislava, 84248 Bratislava, Slovakia

${ }^{9}$ CERN, 1211, Geneva 23, Switzerland

${ }^{10}$ St. Petersburg Electrotechnical University "LETI", 197376 St. Petersburg, Russia

${ }^{11}$ Department of Physics, University of Liverpool, Liverpool, L69 7ZE, United Kingdom

${ }^{12}$ Belgian Nuclear Research Center SCK•CEN, Boeretang 200, B-2400 Mol, Belgium

${ }^{13}$ GSI Helmholtzzentrum für Schwerionenforschung GmbH, Darmstadt 64291, Germany

${ }^{14}$ Center for Nuclear Study (CNS), Graduate School of Science The University of Tokyo, Japan

${ }^{15}$ CSNSM-IN2P3, Université de Paris Sud, Orsay, France

${ }^{16}$ Universität Greifswald, Institut für Physik, 17487 Greifswald, Germany

${ }^{17}$ Institut für Physik, Johannes Gutenberg-Universität Mainz, Mainz, D-55128, Germany

${ }^{18}$ Institut für Kern- und Teilchenphysik, Technische Universität Dresden, Dresden 01069, Germany

(Received 28 January 2020; accepted 21 February 2020; published 20 March 2020)

\begin{abstract}
Hyperfine-structure constants for the $6 s^{2} S_{1 / 2}$ and $6 p^{2} P_{1 / 2}$ atomic states of the $I^{\pi}=11 / 2^{-}$gold isomers ${ }_{177,191,193,195} \mathrm{Au}^{m}$ have been measured at CERN-ISOLDE, using the in-source laser resonance-ionization spectroscopy technique. From the measured hyperfine constants the differences between hyperfine anomalies for these atomic states have been deduced. These differential hyperfine anomaly values have been used to determine the $6 s$-state hyperfine anomaly relative to the stable ${ }^{197} \mathrm{Au}$ with advanced atomic calculations. Magnetic dipole moments for the gold isomers in question have been deduced, taking into account the corresponding relative hyperfine-anomaly values. It has been shown that the commonly used prescription for the extraction of the magnetic moment values for the gold isotopes should be reconsidered. The magnetic moments calculated by this prescription have been reevaluated by properly accounting for the hyperfine anomaly, which is as large as $10 \%$ for several gold isotopes.
\end{abstract}

DOI: 10.1103/PhysRevC.101.034308

\footnotetext{
*barzakh_ae@pnpi.nrcki.ru

${ }^{\dagger}$ Present address: CERN, 1211, Geneva 23, Switzerland.

${ }^{\ddagger}$ Present address: Accelerator Division, TRIUMF, Vancouver BC, Canada V6T 2A3.

${ }^{\S}$ Present address: Centre d'Etudes Nucléaires de BordeauxGradignan, 19 Chemin du Solarium, CS 10120, F-33175 Gradignan, France.

"Present address: Université Paris-Saclay, CNRS/IN2P3, IJCLab, 91405 Orsay, France.
}

\footnotetext{
IPresent address: ARC Centre of Excellence for Engineered Quantum Systems, School of Physics, The University of Sydney, NSW 2006, Australia.

Published by the American Physical Society under the terms of the Creative Commons Attribution 4.0 International license. Further distribution of this work must maintain attribution to the author(s) and the published article's title, journal citation, and DOI.
} 


\section{INTRODUCTION}

The magnetic dipole moment $\mu_{A}$ for a nucleus of mass number $A$ and with a spin $I$ can be calculated using the following expression:

$$
\mu_{A}=\mu_{\mathrm{ref}} \frac{I_{A}}{I_{\mathrm{ref}}} \frac{a_{A}}{a_{\mathrm{ref}}},
$$

where $a$ is a magnetic hyperfine constant and the subscript "ref" denotes a reference isotope with known $\mu$ and $a$ values.

However, Eq. (1) is based on a pointlike approximation for the charge and magnetization in the nucleus. The finite size of the nucleus leads to the deviation of the hyperfine constant from the pointlike value $a_{\mathrm{pt}}$. Correspondingly, two parameters $\delta$ and $\varepsilon$ were introduced to account for the charge and magnetization distribution within the finite-size nucleus. They are called the "Breit-Rosenthal" (BR) hyperfine anomaly (HFA) [1,2] and "Bohr-Weisskopf" (BW) hyperfine anomaly [3], respectively. To account for these two effects, the hyperfine constant $a$ should be expressed as

$$
a=a_{\mathrm{pt}}(1+\delta)(1+\varepsilon),
$$

where $\delta$ and $\varepsilon$ are small compared to unity. These corrections are isotope dependent and can be experimentally observed as small deviations of the $a$-factor ratios between different isotopes from the ratios of their magnetic moments. This deviation, known as a relative hyperfine anomaly (RHFA), is given by

$$
\begin{aligned}
{ }^{\mathrm{ref}} \Delta^{A} & \equiv \frac{a_{\mathrm{ref}}}{a_{A}} \frac{\mu_{A}}{I_{A}} \frac{I_{\mathrm{ref}}}{\mu_{\mathrm{ref}}}-1={ }^{\mathrm{ref}} \Delta_{\mathrm{BW}}^{A}+{ }^{\mathrm{ref}} \Delta_{\mathrm{BR}}^{A} \\
& \approx\left(\varepsilon_{\mathrm{ref}}-\varepsilon_{A}\right)+\left(\delta_{\mathrm{ref}}-\delta_{A}\right) .
\end{aligned}
$$

For heavy atoms $\Delta_{\mathrm{BR}}$ is expected to be negligible compared to $\Delta_{\mathrm{BW}}\left({ }^{A} \Delta_{\mathrm{BR}}^{A+2} \approx 10^{-4}\right.$ in the gold region [4], whereas in all cases relevant to the present work $\Delta_{\mathrm{BW}} \geqslant 10^{-2}$; see below). Accordingly, in the following discussion we will ignore the $\Delta_{\mathrm{BR}}$ contribution to the RHFA.

Equation (1) needs to be modified to account for the finite size of the nucleus:

$$
\begin{aligned}
\mu_{A} & =\mu_{\mathrm{ref}} \cdot \frac{I_{A}}{I_{\mathrm{ref}}} \cdot \frac{a_{A}}{a_{\mathrm{ref}}} \cdot\left(1+{ }^{\mathrm{ref}} \Delta^{A}\right) \\
& \approx \mu_{\mathrm{ref}} \cdot \frac{I_{A}}{I_{\mathrm{ref}}} \cdot \frac{a_{A}}{a_{\mathrm{ref}}} \cdot\left(1+\varepsilon_{\mathrm{ref}}-\varepsilon_{A}\right) .
\end{aligned}
$$

To determine the RHFA from Eq. (3) one should have independent values for the nuclear magnetic moments $\mu$ and $a$ constants of the pair of isotopes under study, measured with high precision. Therefore, the available RFHA data are restricted mainly to stable and long-lived nuclei [5].

The lack of systematic experimental data for RHFA far from stability hampers the development of a theoretical analysis of this important nuclear observable, which is sensitive to the nuclear configuration [6]. One of the aims of the present study is to extend our knowledge of RHFA to short-lived isotopes, by the application of the method proposed in Refs. [7,8] in combination with advanced atomic calculations (see details in Secs. III and V below).

Experimentally measured RHFA values, ${ }^{\text {ref }} \Delta^{A}$, are usually within the range of $10^{-2}-10^{-4}$ [5]. For short-lived nuclei,
${ }^{\text {ref }} \Delta^{A}$ in Eq. (4) is typically neglected and the uncertainty of the extracted magnetic moments is increased by $\approx 1 \%$. In most cases this approach is acceptable in view of the experimental uncertainties and nuclear physics inferences.

However, there is a marked exception in gold isotopes, where ${ }^{197} \Delta^{198}=8.53(8) \%$ was reported in Ref. [9] (see also the large RHFA of the order of 3\% in silver isotopes [5]). Such a large anomaly demands the estimation of the RHFA to obtain reliable magnetic moment values for gold isotopes far from stability. Ekström et al. [9] proposed a procedure (referred to as the standard prescription in the following text) to account for the large RHFA in gold isotopes, which was used in all subsequent works devoted to hyperfine-structure (hfs) measurements in gold isotopes. However, this procedure is not well grounded, as will be shown in Sec. II of the present study. Instead of using the standard prescription, we have deduced the magnetic moment values by Eq. (4) owing to the determination of the RHFA for the gold isotopes in question.

The investigation presented in this paper is part of an experimental campaign at the ISOLDE facility (CERN) aimed at nuclear decay- and laser-spectroscopy studies of the neutrondeficient gold isotopes. Partial results for ${ }^{177,179} \mathrm{Au}$ were reported in Ref. [10]. In the present work we report the study of the hyperfine structure of the $I^{\pi}=11 / 2^{-}$isomers in the $177,191,193,195 \mathrm{Au}$ isotopes. With the RHFAs determined in the present work for the first time, reliable magnetic moments for the selected gold isomers have been derived and some of the previously determined values have been reconsidered. The deduced magnetic moments have enabled us to trace the evolution of the $g$ factor $(g=\mu / I)$ for the $\pi h_{11 / 2}$ orbital from $N=82$ to $N=126$.

\section{THE STANDARD PROCEDURE TO ACCOUNT FOR RHFA FOR GOLD ISOTOPES ("STANDARD PRESCRIPTION")}

Ekström et al. [9] proposed a procedure to evaluate the magnetic moments from the measured magnetic hyperfine constants $a$ in gold isotopes. This procedure is based on the empirical Moskowitz-Lombardi (ML) rule [11] which was successfully applied to odd-neutron mercury isotopes $(A=$ 193 - 203). It was found that the experimental RHFA data for a number of the mercury isotopes, including the neutron shellmodel states $p_{1 / 2}, p_{3 / 2}, f_{5 / 2}$, and $i_{13 / 2}$, are well reproduced by the simple relation (neglecting the BR anomaly) [11]:

$$
\varepsilon=\frac{ \pm \alpha}{\mu}, \quad I=l \pm \frac{1}{2},
$$

where $l$ is the orbital momentum and $\alpha$ is a constant ( $\alpha=$ 0.01 ) independent of the nuclear state.

This empirical approach was justified theoretically by Fujita and Arima [6]. They showed that the HFA may be presented in the following form:

$$
\varepsilon=c_{1}+\frac{\alpha}{\mu} .
$$

For mercury isotopes $c_{1} \approx 0.01$ and $\alpha= \pm 0.012$ [6]. According to Ref. [6] the constants $c_{1}$ and $\alpha$ are strongly state dependent in contrast to the ML rule. Due to the similarity 
of the $\alpha$ values and the cancellation of the $c_{1}$ term in the expression for the RHFA [ $\varepsilon_{\text {ref }}-\varepsilon_{A}$, Eq. (3)], the use of Eq. (5) or (6) gives nearly identical results for the RHFA, whereas absolute HFA values $\left(\varepsilon_{A}\right)$ are different due to the presence of the additional $c_{1}$ term in the theoretical relation. It is impossible to confirm or disprove the necessity of this term experimentally, since only the difference between HFA values (RHFA) can be measured.

The ML rule (with $\alpha=0.012$ ) also holds true for ${ }^{196-199} \mathrm{Au}$ [9]. Using Eq. (5), Ekström et al. deduced an absolute value of $\varepsilon_{197}=8.21 \%$ for the HFA of ${ }^{197} \mathrm{Au}$ [9]. A calculation of the quantity $a_{\mathrm{pt}} I / \mu$ using Eq. (2) with this absolute HFA value gives $a_{\mathrm{pt}} I / \mu=29005 \mathrm{MHz} / \mu_{N}$ Correspondingly, the standard prescription for extracting the magnetic moment value from the measured hfs constant of the gold isotope is

$$
\mu=\frac{a I}{29005} \pm 0.012 \mu_{N}, \quad \varepsilon=\mp \frac{0.012}{\mu}, \quad I=l \pm 1 / 2
$$

for single-particle shell-model states in even- $N$ gold isotopes and

$$
\mu_{A}=\frac{a_{A} I_{A}}{29005\left(1+\varepsilon_{A}\right)}
$$

in the general case. The derivation of Eqs. (7) and (8) is given in detail in Ref. [9]. Since $\varepsilon_{A}$ values for the radioactive gold isotopes are unknown, one usually omits it in Eq. (8), while the experimental error accounts for its uncertainty: $\left|\varepsilon_{A}\right|<$ $0.012 /|\mu|$ for odd-even nuclei and $\left|\varepsilon_{A}\right|<0.024 /|\mu|$ for oddodd isotopes $[9,12]$. Note that this uncertainty estimation is also based on the ML rule $[9,12]$.

However, there are several not well justified assumptions which underlie the standard prescription.

1. There is an indeterminacy in the absolute value of $\varepsilon_{197}$. Reasonable assumptions regarding the value of $c_{1}$ in Eq. (6) [6] lead to an additional $2 \%$ uncertainty in the $a_{\mathrm{pt}} / g$ ratio and, correspondingly, in associated $\mu$ values. One should also take into account the uncertainty in $\varepsilon_{A}$ when using Eq. (8) instead of Eq. (7), which leads to an additional uncertainty of the order of $2 \%$ in the $\mu$ value.

2. The standard prescription is based on the ML rule. However, it was shown that this rule is not universal and one should take care with its application [13-15].

Thus, the reliability of the $\mu$ values and their uncertainties obtained by the standard prescription, is questionable and it is necessary to find another procedure to account for the large RHFA in gold isotopes. In the present work such a procedure has been applied. The hfs parameters of the $I^{\pi}=11 / 2^{-}$gold isomers ${ }^{177,191,193,195} \mathrm{Au}^{m}$, measured in the present work, have been analyzed and the respective RHFA values have been obtained using the results of the atomic calculations. In the Appendix the same procedure has been applied for some previously studied gold isotopes.

\section{DIFFERENTIAL HYPERFINE ANOMALY AND A PROCEDURE FOR RHFA EXTRACTION}

The hyperfine anomaly reveals itself in the change in the ratio of the magnetic hfs constants for different atomic states with quantum numbers $n_{1} l_{1}$ and $n_{2} l_{2}$. This ratio, $a_{n_{1} l_{1}}^{A} / a_{n_{2} l_{2}}^{A}$, depends on the atomic mass number $A$, because different atomic states differ in sensitivity to the nuclear magnetization distribution. This change can be related to the difference of the corresponding RHFA values by introducing differential HFA (DHFA):

$$
\begin{aligned}
{ }_{n_{1} l_{1}}^{A_{1}} \Delta_{n_{2} l_{2}}^{A_{2}} & \equiv \frac{\left(a_{n_{1} l_{1}}^{A_{1}} / a_{n_{2} l_{2}}^{A_{1}}\right)}{\left(a_{n_{1} l_{1}}^{A_{2}} / a_{n_{2} l_{2}}^{A_{2}}\right)}-1=\frac{1+{ }^{A_{1}} \Delta^{A_{2}}\left(n_{1} l_{1}\right)}{1+{ }^{A_{1}} \Delta^{A_{2}}\left(n_{2} l_{2}\right)}-1 \\
& \approx{ }^{A_{1}} \Delta^{A_{2}}\left(n_{1} l_{1}\right)-{ }^{A_{1}} \Delta^{A_{2}}\left(n_{2} l_{2}\right) .
\end{aligned}
$$

It was shown in Refs. $[3,16]$ that the BW anomaly may be represented in the single-particle nuclear shell model and oneelectron approximation as the product of two factors, one of them being dependent only on the atomic structure, the second being dependent only on the nuclear properties. This atomicnuclear factorization was confirmed in Refs. $[6,17,18]$, where more-refined atomic and nuclear models were used.

Due to this atomic-nuclear factorization, the ratio

$$
\eta_{n_{1} l_{1}, n_{2} l_{2}} \equiv \frac{{ }^{A_{1}} \Delta^{A_{2}}\left(n_{1} l_{1}\right)}{A_{1} \Delta^{A_{2}}\left(n_{2} l_{2}\right)}
$$

should be independent of $A$ and is determined solely by the electronic wave function. The factor $\eta$ can be determined experimentally for stable or long-lived nuclei where independent values of the magnetic moments are available. When the corresponding data are missing, this factor can be calculated by using advanced atomic approaches. With a known $\eta$ factor, the RHFA value needed for the magnetic moment evaluation [see Eq. (4)] is deduced from the measured DHFA,

$$
{ }^{A_{1}} \Delta^{A_{2}}\left(n_{1} l_{1}\right)=\frac{{ }_{n_{2} l_{2}}^{A_{1}} \Delta_{n_{1} l_{1}}^{A_{2}}}{1 / \eta_{n_{1} l_{1}, n_{2} l_{2}}-1-{ }_{n_{2} l_{2}}^{A_{1}} \Delta_{n_{1} l_{1}}^{A_{2}}} .
$$

To summarize, it is possible to determine the RHFA without having independent measurements of magnetic moments, by measuring magnetic hyperfine constants for different atomic levels and deducing the corresponding $\eta$ factor by either atomic calculations or (if possible) from the hfs measurements for isotopes with a known RHFA.

This approach was first implemented for the gallium isotopes [7] (see also more general considerations on using DHFA for anomaly analysis in Ref. [8]). Subsequently, the same procedure was applied for thallium [14] and bismuth [19] nuclei. It is worth emphasizing, that in this way one can determine the RHFA for nuclei far from stability. Thus, this procedure allows us to gain insight into how the nuclear magnetization distribution of ground and isomeric states changes from one isotope to another.

\section{EXPERIMENTAL DETAILS}

The present data originate from the same experiment as described in Ref. [10]. The gold nuclei were produced in spallation reactions induced by the $1.4-\mathrm{GeV}$ proton beam 


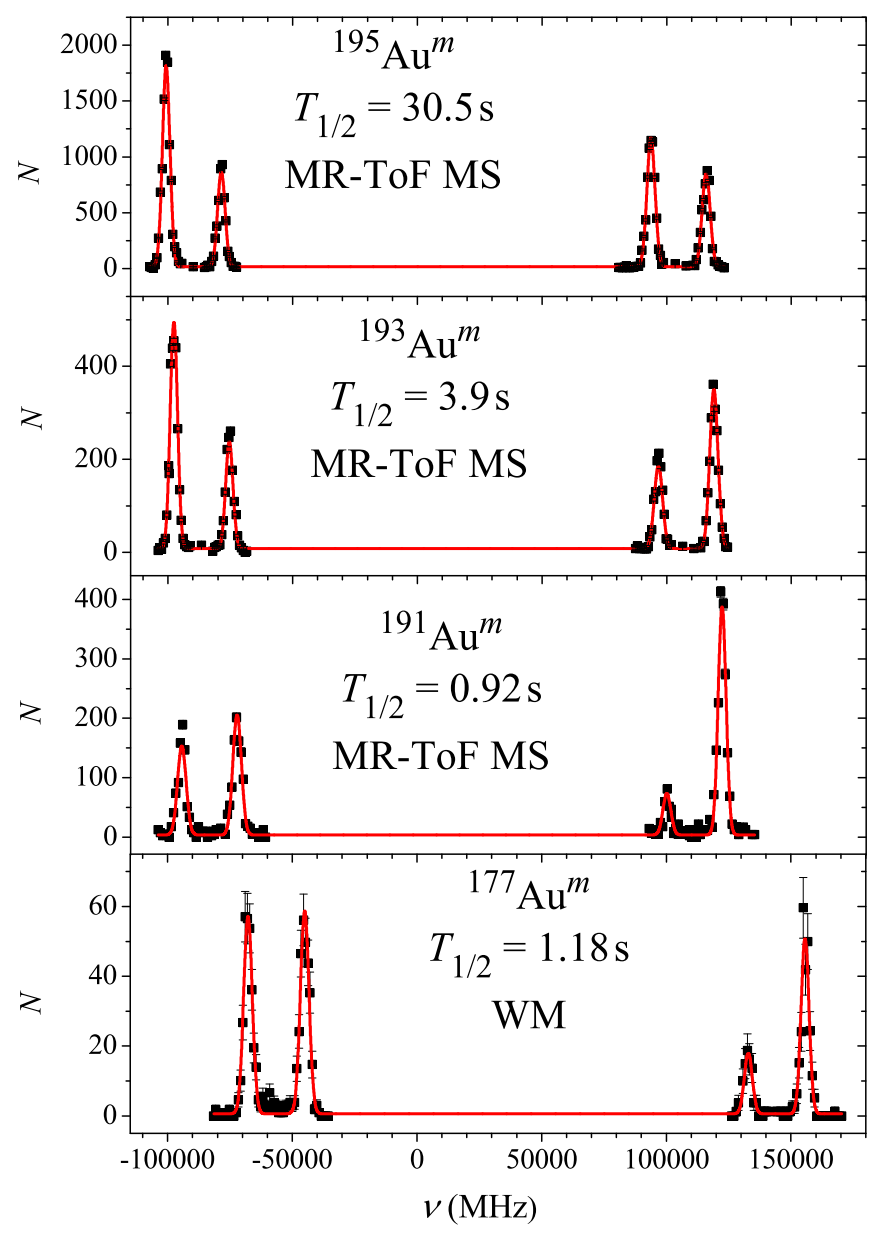

FIG. 1. The hfs spectra of selected Au isomers. The half-life and the method of the photoion current monitoring are shown for each isomer. The solid lines depict the Voigt-profile fit to the data. The zero point on the frequency scale corresponds to a wave number of $37358.90 \mathrm{~cm}^{-1}$.

from the CERN PS Booster, impinging on a $50 \mathrm{~g} \mathrm{~cm}^{-2}$ thick $\mathrm{UC}_{x}$ target. The reaction products diffused out of the high temperature target $(T \approx 2500 \mathrm{~K}$ ) and effused as neutral atoms into the hot cavity of the ion source. Inside the cavity, the gold atoms were selectively ionized by the ISOLDE Resonance Ionization Laser Ion Source installation (RILIS) [20,21], with the laser beams frequency tuned to the three-step gold ionization scheme [22]. The ions were then extracted from the cavity using a $30-\mathrm{kV}$ electrostatic potential and separated according to their mass-to-charge ratio by the General Purpose Separator of ISOLDE [23].

The mass-separated beam was then delivered to either the ISOLTRAP Multi-Reflection Time-of-Flight Mass Spectrometer (MR-ToF MS) [24] or the Windmill (WM) decay station $[25,26]$, for photoion monitoring during wavelength scans. Details of the scanning procedures can be found in Refs. [25,26]. The hfs measurements were made upon the 267.7-nm atomic transition in gold $\left(6 s^{2} S_{1 / 2} \rightarrow 6 p^{2} P_{1 / 2}\right)$, by scanning a frequency-tripled titanium sapphire laser in a narrowband mode (bandwidth of $\approx 600 \mathrm{MHz}$ before tripling). Two broadband dye lasers (bandwidth of $\approx 20 \mathrm{GHz}$ ) were
TABLE I. Experimental results for $11 / 2^{-}$gold isomers.

\begin{tabular}{llll}
\hline \hline$A$ & $a_{6 s}^{A}(\mathrm{MHz})$ & \multicolumn{1}{c}{$\left(a_{6 p}^{A} / a_{6 s}^{A}\right)$} & ${ }_{6 p}^{197} \Delta_{6 s}^{A}(\%)$ \\
\hline 177 & $33414(34)$ & $0.1142(9)$ & $-7.7(8)$ \\
189 & $32625(42)^{\mathrm{a}}$ & $0.1122(11)^{\mathrm{a}}$ & $-6.0(10)$ \\
191 & $32424(30)$ & $0.1145(9)$ & $-7.9(8)$ \\
193 & $32391(30)$ & $0.1141(7)$ & $-7.6(6)$ \\
195 & $32372(46)$ & $0.1141(9)$ & $-7.5(8)$ \\
\hline \hline
\end{tabular}

${ }^{\text {a Reference [12]. }}$

used for the second and third excitation steps. Examples of the experimental hfs spectra for the studied $I^{\pi}=11 / 2^{-}$gold isomers are shown in Fig. 1.

The positions of the hyperfine components as a function of the scanning laser frequency are determined by the formula:

$$
v^{F, F^{\prime}}=v_{0}+a_{6 p} \frac{K^{\prime}}{2}-a_{6 s} \frac{K}{2},
$$

where $v_{0}$ is the centroid frequency of the hfs, the prime symbol denotes the upper level of the atomic transition, $K=F(F+$ 1) $-I(I+1)-J(J+1), F$ is the total angular momentum of the atomic level, $I$ and $J$ are the nuclear spin and the angular momentum for the electronic state, respectively, and $a_{n l}$ is the magnetic hyperfine coupling constant for the atomic level with the quantum numbers $n$ and $l$. For brevity throughout the paper indices $6 s$ and $6 p$ will be related to the $6 s^{2} S_{1 / 2}$ and $6 p^{2} P_{1 / 2}$ states, respectively.

In Table I the experimental hfs constants, $a_{6 s}$, and $a$ constants ratios for the $11 / 2^{-}$gold isomers are presented along with the DHFA values ${ }_{6 p}^{197} \Delta_{6 s}^{A}$, calculated by Eq. (9). To convert the latter to RHFA one should know the $\eta_{6 s, 6 p}$ factor [see Eq. (11)]. Unfortunately, there are no experimental data on $a_{6 p}^{A} / a_{6 s}^{A}$ for ${ }^{196,198,199} \mathrm{Au}$ where the RHFA values were determined independently. Therefore it is impossible to deduce a pure experimental value of the $\eta_{6 s, 6 p}$ factor. Accordingly, we used advanced atomic calculations to determine its value.

\section{CALCULATION OF THE RHFA-VALUES RATIO}

For completeness, both BR and BW anomalies in gold atom were calculated, although the contribution of the former to RHFA is much less than the contribution of the latter.

According to Refs. [27,28], the BR correction can be presented in the following form:

$$
\delta=b_{N}\left(R_{C} / \lambda_{C}\right)^{\chi}, \quad \chi=2 \sqrt{1-(\alpha Z)^{2}}-1,
$$

where $\lambda_{C}$ is the Compton wavelength of the electron $\lambda_{C}=$ $\hbar /\left(m_{e} c\right), \alpha$ is the fine structure constant, $Z$ is a nuclear charge, $R_{C}$ is a nuclear charge radius in the homogeneously charged ball approximation, and $b_{N}$ is a dimensionless parameter (we use atomic units $\hbar=e=m_{e}=1$ throughout this section).

Keeping in mind the atomic-nuclear factorization approximation (see Sec. III), the BW correction can be presented by the similar relation $[27,28]$ :

$$
\varepsilon=b_{M} d_{\mathrm{nuc}}\left(R_{M} / \lambda_{C}\right)^{\chi},
$$


TABLE II. Results of calculations for the $6 s^{2} S_{1 / 2}$ and $6 p^{2} P_{1 / 2}$ levels of gold atom.

\begin{tabular}{|c|c|c|c|c|c|}
\hline \multirow[b]{2}{*}{ Method } & \multicolumn{2}{|c|}{$6 s_{1 / 2}$} & \multicolumn{2}{|c|}{$6 p_{1 / 2}$} & \multirow[b]{2}{*}{$\eta_{6 s, 6 p}$} \\
\hline & $b_{N}$ & $b_{M}$ & $b_{N}$ & $b_{M}$ & \\
\hline DHF & 1.273 & 0.243 & 0.340 & 0.065 & 3.73 \\
\hline RPA & 1.213 & 0.241 & 0.324 & 0.056 & 4.31 \\
\hline $\mathrm{RPA}+\mathrm{MBPT}$ & 1.204 & 0.238 & 0.254 & 0.059 & 4.05 \\
\hline $\mathrm{RPA}+\mathrm{CC}$ & 1.118 & 0.238 & 0.236 & 0.058 & 4.08 \\
\hline
\end{tabular}

where $R_{M}$ is a nuclear magnetization radius, parameter $d_{\text {nuc }}$ takes into account the change of the nuclear magnetization distribution for nuclear states with different spins and configurations, and $b_{M}$ is a dimensionless parameter. In the case of a pure shell-model configuration, the parameter $d_{\text {nuc }}$ can be calculated in the framework of the single-particle shell model [29]. This nuclear factor is defined so that $d_{\text {nuc }}=0$ corresponds to a pointlike magnetic dipole in the center of the nucleus and $d_{\text {nuc }}=1$ corresponds to a homogeneously magnetized ball of radius $R_{M}$.

The magnetic hfs constant $a$ can be calculated at different values of $R_{C}$ and $R_{M}$. The dependences of $a$ on $R_{C}$ and $R_{M}$ give parameters $b_{N}$ and $b_{M}$ in accordance with Eqs. (2), (13), and (14).

The magnetic hfs constants were calculated for several low-lying levels in the gold atom. We treated gold as a system with one valence electron above the core $\left[1 s^{2} \ldots 5 p^{6} 4 f^{14} 5 d^{10}\right]$, therefore, we did not consider levels which correspond to excitations from the $5 d$ shell. We used many-body perturbation theory (MBPT) and coupled cluster (CC) approximations on top of the Hartree-Fock-Dirac method (DHF) [30-33]. Correlation corrections to the hyperfine interaction vertex were included within the random phase approximation (RPA) with self-energy correction [34] and structural radiation correction [35]. The results of the calculations by different methods are presented in Table II.

Using the calculated $b_{N}$ value, we estimated BR contribution to RHFA for the most neutron-deficient nucleus in question, ${ }^{177} \mathrm{Au}^{m}$, by Eq. (13): ${ }^{197} \Delta_{\mathrm{BR}}^{177} \approx 10^{-3}$. For heavier nuclei the BR correction is even smaller [see Eq. (13)]. This result substantiates the neglect of the BR anomaly when considering RHFA for gold nuclei.

As a final value for the $\eta_{6 s, 6 p}$ factor in gold we adopted the mean value of the results obtained in the frameworks of the different approximations (see column 6 of Table II) with the uncertainty covering the dispersion of these results: $\eta_{6 s, 6 p}=4.0(3)$.

To check the method of calculation, we compare the experimental and theoretical $\eta$ values for thallium. In Ref. [36] the hfs constant $a(7 s)$ was measured for ${ }^{203,205} \mathrm{Tl}$ with high accuracy. Combining these results with the data from Refs. [37,38] one obtains: $\eta_{7 s, 6 p}(\mathrm{Tl})=2.84(78)$. This experimental value matches well the result of the theoretical calculation, identical to that used in the present work for gold atom: $\eta_{7 s, 6 p}(\mathrm{Tl})=$ $3.0(3)$.

\section{HYPERFINE ANOMALY IN 11/2- GOLD ISOMERS}

In Table III and Fig. 2 the RHFA values for the selected $I^{\pi}=11 / 2^{-}$gold isomers are presented. The hyperfine structure of ${ }^{189} \mathrm{Au}^{m}$ was not measured in the present work and ${ }^{197} \Delta^{A}(6 s)$ value for ${ }^{189} \mathrm{Au}^{m}$ was calculated using the data from Ref. [12]. One can see that the ${ }^{197} \Delta^{A}(6 s)$ value for ${ }^{177,191,193,195} \mathrm{Au}^{m}$ is constant within uncertainties. However, the value for ${ }^{189} \mathrm{Au}^{m}$ differs by just over $1.5 \sigma$, although there are no peculiarities in its nuclear structure compared to other $I^{\pi}=11 / 2^{-}$gold isomers, which could explain this deviation. Correspondingly, ${ }^{197} \Delta^{189 m}(6 s)$ was not taken into account in the calculations of the mean value of the RHFA for the $I^{\pi}=11 / 2^{-}$gold isomers. This weighted mean value, ${ }^{197} \Delta^{(I=11 / 2)}(6 s)=0.1134(58)$, was used to deduce magnetic moments by Eq. (4) (see column 4 in Table III).

Having experimental values of RHFA, one can check the applicability of the ML rule. According to Eq. (5), ${ }^{197} \Delta^{191 m}(6 s ; \mathrm{ML})=0.084$. Thus, the ML rule markedly underestimates the RHFA for the $11 / 2^{-}$gold isomers. This is clearly seen from the comparison of the magnetic moments calculated by the standard prescription and those deduced by Eq. (4) (see Table III).

In view of the failure of the ML rule in the RHFA description of the $11 / 2^{-}$gold isomers, the application of this rule (and, in particular, the standard prescription) to account for the hyperfine anomaly in isotopes of gold becomes questionable.

TABLE III. RHFA, ${ }^{197} \Delta^{A}(6 s)$, and magnetic moments for $I^{\pi}=11 / 2^{-}$gold isomers.

\begin{tabular}{lcclcl}
\hline \hline$A$ & ${ }^{197} \Delta^{A}(6 s)(\%)$ & $\mu\left(\mu_{N}\right)^{\mathrm{a}}$ & $\mu\left(\mu_{N}\right)^{\mathrm{b}}$ & $\mu\left(\mu_{N}\right)^{\mathrm{c}}$, literature & References, methods \\
\hline 177 & $11.4(14)$ & $6.519(38)$ & $6.348(6)$ & & \\
189 & $8.6(16)$ & $6.365(38)$ & $6.198(8)$ & $6.17(15)$ & [42], NMR/ON \\
& & & & $6.186(20)^{\mathrm{d}}$ & {$[12], \mathrm{hfs}$} \\
191 & $11.7(14)$ & $6.326(37)$ & $6.160(6)$ & $6.6(6)$ & {$[43], \mathrm{NO}$} \\
193 & $11.2(11)$ & $6.320(37)$ & $6.154(6)$ & $6.18(9)$ & {$[44], \mathrm{NMR} / \mathrm{ON}$} \\
195 & $11.2(14)$ & $6.316(37)$ & $6.150(9)$ & $6.18(9)$ & {$[39], \mathrm{NMR} / \mathrm{ON}$} \\
\hline \hline
\end{tabular}

${ }^{\mathrm{a} C}$ Calculated by Eq. (4) at ${ }^{197} \Delta^{(I=11 / 2)}(6 s)=0.1134(58)$.

${ }^{\mathrm{b} C a l c u l a t e d}$ by Eq. (7) (the standard prescription). Note, that the uncertainties do not include contribution from the indeterminacies of the standard prescription $(\approx 4 \%$; see text for details).

${ }^{\mathrm{c}}$ Note, that the NMR/ON results should be reconsidered (see text).

${ }^{\mathrm{d}}$ Calculated by Eq. (8) with $\varepsilon_{A}=0$ and the increase of the uncertainty by $0.012 \mu_{N}$. 


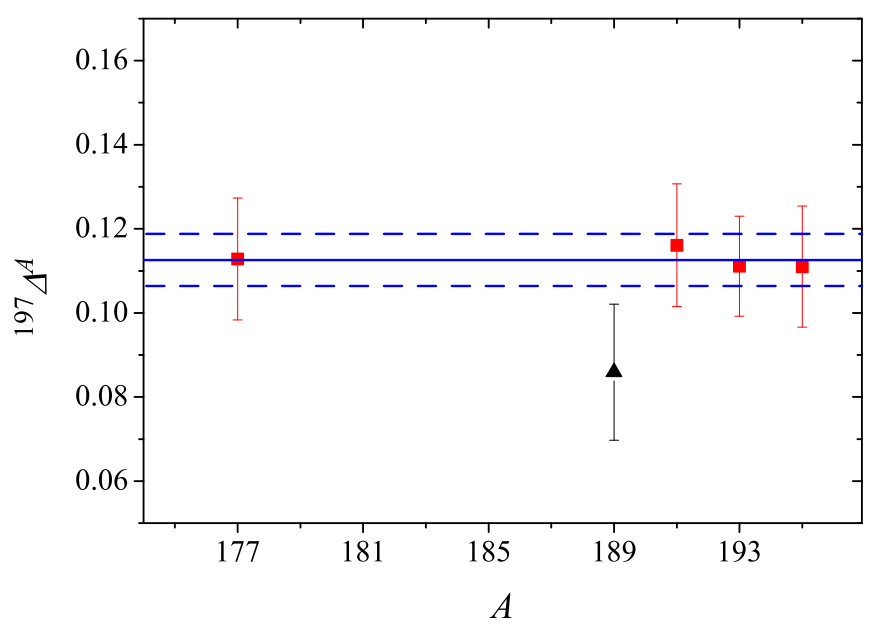

FIG. 2. Relative hyperfine anomaly for $11 / 2^{-}$gold isomers. Squares - present work, triangle $(A=189)$ - RHFA calculated by Eqs. (9)-(11) with the data from Ref. [12]. Lines-weighted mean value for $A=177,191,193,195$ with the corresponding error bars.

Thus, nearly all previously derived magnetic moments in gold nuclei should be recalculated with correct accounting of the RHFA or, at least, the corresponding uncertainties should be revised (see the Appendix).

Magnetic moments of the high-spin gold isomers were measured previously by the method of nuclear magnetic resonance on oriented nuclei (NMR/ON; see Table III). The NMR/ON method relies upon the precise determination of the magnetic hyperfine splitting of radioactive nuclei in a ferromagnetic host lattice. To extract the magnetic moment, one should know the effective magnetic field $B_{\mathrm{hf}}$ seen by a nucleus embedded in a ferromagnetic host. This field depends on the nuclear magnetization distribution over the nuclear volume, which leads to a "NMR/ON" hyperfine anomaly. This anomaly is not equal to the hfs anomaly, due to the noncontact hyperfine field which should be taken into account in the analysis of the NMR/ON experiments. The difference between these anomalies is believed to be small $(\approx 10 \%$ according to the estimation in Refs. [39,40]), and to determine $B_{\mathrm{hf}}$ and thus the magnetic moments, the RHFA correction was taken into account in the corresponding publications. In Ref. [39] the ${ }^{198} \Delta^{195 m}(6 s)$ value was empirically estimated using the ML rule, Eisinger and Jaccarino calculations [41], and strong assumptions on the nuclear configuration in ${ }^{198} \mathrm{Au}$. The result, ${ }^{198} \Delta^{195 m}(6 s)_{\mathrm{emp}}=0.004(15)$, differs noticeably from the value obtained by combining experimental values of ${ }^{197} \Delta^{198}(6 s)$ [9] and ${ }^{197} \Delta^{(I=11 / 2)}(6 s):{ }^{198} \Delta^{195 m}(6 s)_{\text {expt }}=$ ${ }^{197} \Delta^{(I=11 / 2)}(6 s)_{\text {expt }}-{ }^{197} \Delta^{198}(6 s)_{\text {expt }}=0.028(6)$. This means that even when assuming equal "NMR/ON" and hfs anomalies, the results and uncertainties of the NMR/ON measurements for the high-spin gold isomers should be reconsidered.

\section{MAGNETIC MOMENTS OF THE $\pi h_{11 / 2}$ STATES AT $82 \leqslant N \leqslant 126$}

In Fig. 3 the systematics of the $g$ factors of the $\pi h_{11 / 2}$ states is presented. Along with the values for gold isomers deter-

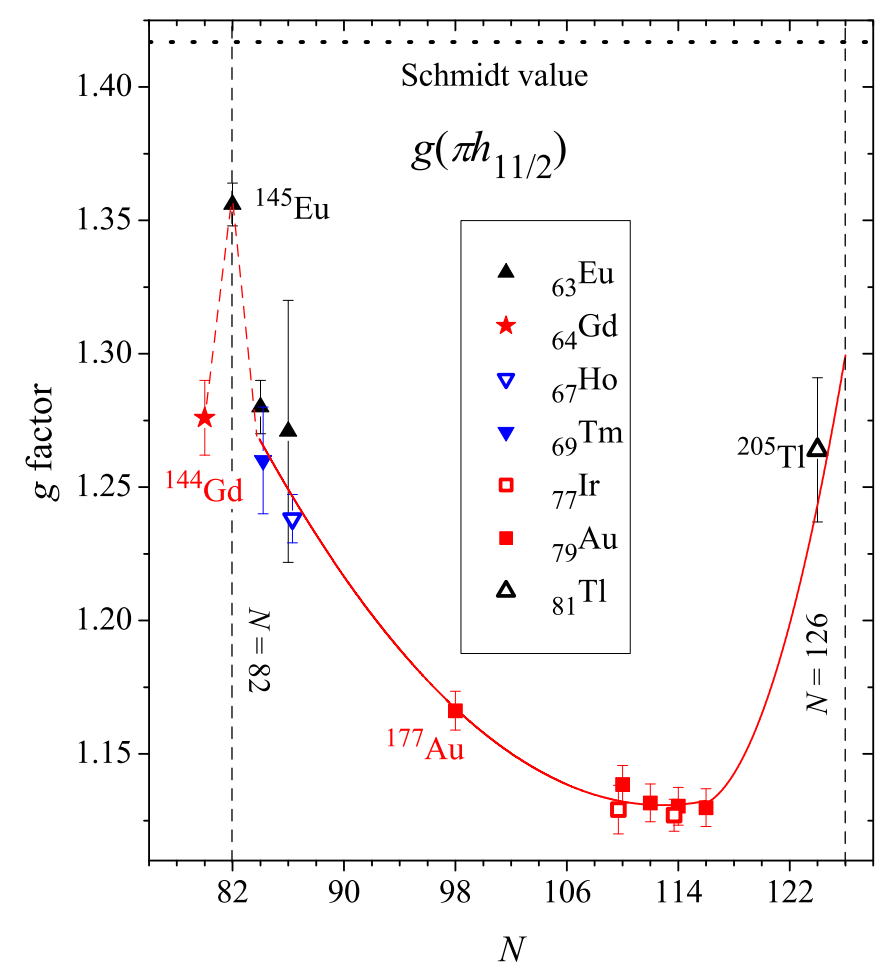

FIG. 3. $g$ factors of the $\pi h_{11 / 2}$ states. The parabolic-type curves are to guide the eye.

mined in the present work, the experimental data for iridium ([44-46] and references therein), europium [47], holmium [48], thulium [49], gadolinium [50], and thallium [51] are shown. In the cases of ${ }^{144} \mathrm{Gd}$ and ${ }^{205} \mathrm{Tl}, g\left(\pi h_{11 / 2}\right)$ values were derived from the measured $g$ factors for $I^{\pi}=10^{+}\left(h_{11 / 2}\right)^{2}$ and $I^{\pi}=25 / 2^{+}\left(7^{-} \otimes h_{11 / 2}\right)$ states, respectively [50,51]. Thus, we have unique systematics of the single-particle $g$ factors, spanning the whole range between the magic neutron numbers, $N=82$ and $N=126$.

For such a wide range of proton number (from $Z=64$ to $Z=81)$, the $g\left(\pi h_{11 / 2}\right)$ values display a very regular $N$ dependence: Starting from the maximal value at the magic number $N=82$, it steeply decreases with the increase of $N$, approaching a constant value at $N>110$. The value for ${ }^{205} \mathrm{Tl}$ demonstrates an increase to higher values, similar to those close to $N=82$, although the $g$ factor for the $\pi h_{11 / 2}$ state in ${ }^{205} \mathrm{Tl}$ was deduced by the additivity relation rather than measured directly [51].

Such a behavior can be explained by the evolution of the first-order core-polarization correction (CP1) to the magnetic moment value [52] due to $v f_{7 / 2} \rightarrow v f_{5 / 2}$ core excitations [47]. The occupation of the $v f_{7 / 2}$ orbital starts at $N=82$ and increases with increasing $N$, resulting in an enhancement of the core polarization and corresponding decrease of the magnetic moment. After the complete filling of the $v f_{7 / 2}$ orbital the CP1 correction and $g$ factor remain constant until the start of the $v f_{5 / 2}$-orbital filling at $N=118$. The population of this orbital leads to a blocking of the states available for the $v f_{7 / 2} \rightarrow v f_{5 / 2}$ excitations and, correspondingly, to a decrease in the $\mathrm{CP} 1$ correction for $g\left(\pi h_{11 / 2}\right)$. 
The existing data support this qualitative interpretation. To substantiate these claims it is important to fill the gaps in the $g\left(\pi h_{11 / 2}\right)$ systematics, namely, to measure magnetic moments for the long-lived $11 / 2^{-}$states in ${ }^{167-173,193-197} \operatorname{Ir}_{90-96,116-120}$, ${ }^{207} \mathrm{Tl}_{126},{ }^{141} \mathrm{Eu}_{78},{ }^{205} \mathrm{Au}_{126}$.

\section{CONCLUSIONS}

Hyperfine structure constants of the $11 / 2^{-}$gold isomers ${ }_{177,191,193,195} \mathrm{Au}^{m}$ have been measured using the 267.7-nm atomic transition. The differential hyperfine anomaly has been determined for these nuclei by the comparison of the ratios of the magnetic hyperfine constants of the ground and excited atomic states for different isotopes and isomers. The obtained DHFA values have been converted to the relative hyperfine anomaly using advanced atomic calculations. Magnetic dipole moments have been deduced taking into account the corresponding RHFA values. The applied method of the RHFA determination can be used for other far-from-stability nuclei.

It has been shown that the commonly used procedure for the extraction of the magnetic moment values for gold isotopes (standard prescription) should be reconsidered. The magnetic moments previously calculated by this procedure have been reevaluated with proper accounting for the hyperfine anomaly. It has also been shown that the empirical Moskowitz-Lombardi rule does not work for the investigated nuclei.

Systematics of the nuclear $g$ factor for the $\pi h_{11 / 2}$ states spanning the whole range between the magic neutron numbers, $N=82$ and $N=126$, can be qualitatively explained by the first-order core-polarization correction with the leading role of the $v f_{7 / 2} \rightarrow v f_{5 / 2}$ core excitations.

\section{ACKNOWLEDGMENTS}

This work was done with support from the European Union's Horizon 2020 Framework research and innovation programme under Grant Agreements No. 654002 (ENSAR2) and No. 665779 (CERN-COFUND), by RFBR according to the Research Project No. 19-02-00005, by grants from the U.K. Science and Technology Facilities Council, by FWOVlaanderen (Belgium), by GOA/2010/010 (BOF KU Leuven), by the Interuniversity Attraction Poles Programme initiated by the Belgian Science Policy Office (BriX network P7/12), by the Slovak Research and Development Agency (Contract No. APVV-18-0268) and the Slovak Grant Agency VEGA (Contract No. 1/0532/17), by the German Federal Ministry of Education and Research (BMBF, Contracts No. 05P12HGCI1, No. 05P15HGCIA, and No. 05P18HGCIA). This project has received funding through the European Union's Seventh Framework Programme for Research and Technological Development under Grant Agreements No. 262010 (ENSAR), No. 267194 (COFUND), and No. 289191 (LA ${ }^{3}$ NET). Y.A.D. and M.G.K. were supported by Foundation for the Advancement of Theoretical Physics and Mathematics "BASIS."

\section{APPENDIX}

According to Eqs. (4) and (9)-(11) to account for the RHFA in the magnetic moments extraction from the hfs constants of the gold isotopes, one should know the $a_{6 s}$ and $a_{6 p}$ hyperfine constants. This is the case for some previously studied gold isotopes. References with the relevant information are shown in column 7 of Table IV. New values for magnetic moments calculated by Eq. (4) with ${ }^{197} \Delta^{A}(6 s)$ derived by Eqs. (9)-(11) (see column 5) are presented in column 6. Literature values of magnetic moments with the corresponding references are shown in columns 3 and 4.

In Ref. [53] the hfs of the $5 d^{9} 6 s^{2}{ }^{2} D_{3 / 2} \rightarrow 5 d^{10} 6 p^{2} P_{1 / 2}$ transition in gold was studied. In the magnetic moment evaluation, the hyperfine anomaly was neglected because, as the authors state, there is no $s$ electron involved in the hyperfine interaction of either the ${ }^{2} D_{3 / 2}$ or the ${ }^{2} P_{1 / 2}$ state. However, the RHFA in the ${ }^{2} P_{1 / 2}$ state of gold is not negligible (see Sec. V) - for example, for ${ }^{198} \mathrm{Au}$ it is larger than $2 \%$. On the other hand, we cannot a priori neglect the RHFA in the ${ }^{2} D_{3 / 2}$ state only in view of its leading $5 d^{9} 6 s^{2}$ configuration without an unpaired $s$ electron. For example, in thallium the RHFA value in the $6 p P_{3 / 2}$ state without an unpaired $s$ electron in the leading configuration proves to be 5 times larger than that for the $7 s S_{1 / 2}$ state $[36,56]$ due to the configuration mixing (see also the unexpectedly large RHFA for the $6 p^{3}{ }^{4} S_{3 / 2}$ state in bismuth [19]). The coincidence of the recalculated $\mu$ values with that from Ref. [53] gives the opportunity

TABLE IV. Comparison of the literature data for the magnetic moments, $\mu_{\text {lit }}$, of some gold isotopes with the values recalculated using Eqs. (4) and (9)-(11), $\mu_{\text {recalc. }}$.

\begin{tabular}{|c|c|c|c|c|c|c|}
\hline$A$ & $I$ & $\mu_{\mathrm{lit}}\left(\mu_{N}\right)$ & Refs. for $\mu_{\text {lit }}$ & ${ }^{197} \Delta^{A}(6 s)(\%)$ & $\mu_{\text {recalc }}\left(\mu_{N}\right)$ & Refs. for $a_{6 s}$ and $a_{6 p}$ \\
\hline 194 & 1 & $0.0763(13)$ & {$[53]$} & $1.8(33)$ & $0.0754(25)$ & {$[53,54]$} \\
\hline 193 & $3 / 2$ & $0.1396(5)$ & [53] & $-0.5(11)$ & $0.1398(15)$ & {$[53,55]$} \\
\hline 189 & $1 / 2$ & $0.494(14)$ & {$[12]$} & $9.4(59)$ & $0.499(27)$ & [12] \\
\hline $189 \mathrm{~m}$ & $11 / 2$ & $6.186(20)^{\mathrm{a}}$ & {$[12]$} & $8.6(16)$ & $6.365(38)$ & {$[12]$} \\
\hline 187 & $1 / 2$ & $0.535(15)$ & [12] & $12.7(84)$ & $0.557(41)$ & [12] \\
\hline 185 & $5 / 2$ & $2.170(17)$ & {$[12]$} & $9.4(30)$ & 2.193(61) & {$[12]$} \\
\hline
\end{tabular}

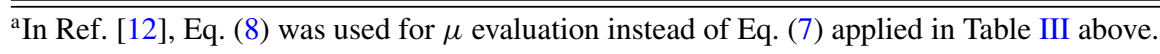


for the first time to confirm the assumption of the small RHFA in ${ }^{194,193,191} \mathrm{Au}$ for the ${ }^{2} D_{3 / 2}$ states of gold. However, the uncertainties ascribed in Ref. [53] to the $\mu$ values are underestimated.

In Ref. [12] the standard prescription [Eq. (8) with $\varepsilon_{A}=$ $0]$ was applied to derive magnetic moments. An additional uncertainty $\left(0.012 \mu_{N}\right.$ for odd- $A$ and $0.024 \mu_{N}$ for even- $A$ isotopes) was introduced with the reference to the ML rule, to account for neglected RHFA. However, as shown in Secs. II and VI, the standard prescription and ML rule are not well justified for all gold nuclei. Correspondingly, the uncertainties quoted in Ref. [12] are underestimated and the addi- tional uncertainty stemming from the indeterminacy of the standard prescription should be taken into account (4\%, see Sec. II).

The magnetic moments of ${ }^{183} \mathrm{Au},{ }^{184} \mathrm{Au}^{g},{ }^{184} \mathrm{Au}^{m}$ were determined by the standard prescription with $\varepsilon=0[57,58]$ but the data on $a_{6 p}$ are missing and it is impossible to apply our procedure to calculate the RHFA. Keeping in mind the possible failure of the standard prescription for these nuclei, the uncertainties ascribed in Refs. [57,58] to the magnetic moment values should be increased by $4 \%$, namely, $\mu\left({ }^{183} \mathrm{Au}\right)=1.97(10) \mu_{N}, \mu\left({ }^{184} \mathrm{Au}^{g}\right)=2.07(10) \mu_{N}$, $\mu\left({ }^{184} \mathrm{Au}^{m}\right)=1.44(7) \mu_{N}$.
[1] J. E. Rosenthal and G. Breit, Phys. Rev. 41, 459 (1932).

[2] M. F. Crawford and A. K. Schawlow, Phys. Rev. 76, 1310 (1949).

[3] A. Bohr and V. F. Weisskopf, Phys. Rev. 77, 94 (1950).

[4] H. J. Rosenberg and H. H. Stroke, Phys. Rev. A 5, 1992 (1972).

[5] J. R. Persson, At. Data Nucl. Data Tables 99, 62 (2013).

[6] T. Fujita and A. Arima, Nucl. Phys. A 254, 513 (1975).

[7] V. J. Ehlers, Y. Kabasakal, H. A. Shugart, and O. Tezer, Phys. Rev. 176, 25 (1968).

[8] J. R. Persson, Eur. Phys. J. A 2, 3 (1998).

[9] C. Ekström, L. Robertsson, S. Ingelman, G. Wannberg, and I. Ragnarsson, Nucl. Phys. A 348, 25 (1980).

[10] J. G. Cubiss et al., Phys. Lett. B 786, 355 (2018).

[11] P. A. Moskowitz and M. Lombardi, Phys. Lett. B 46, 334 (1973).

[12] K. Wallmeroth, G. Bollen, A. Dohn, P. Egelhof, U. Krönert, M. J. G. Borge, J. Campos, A. Rodriguez Yunta, K. Heyde, C. De Coster, L. Wood, H.-J. Kluge, and the ISOLDE Collaboration, Nucl. Phys. A 493, 224 (1989).

[13] J. R. Persson, Hyperfine Interact. 162, 139 (2005).

[14] A. E. Barzakh, L. Kh. Batist, D. V. Fedorov, V. S. Ivanov, K.A. Mezilev, P. L. Molkanov, F. V. Moroz, S. Yu. Orlov, V. N. Panteleev, and Yu. M. Volkov, Phys. Rev. C 86, 014311 (2012).

[15] N. Frömmgen et al., Eur. Phys. J. D 69, 164 (2015).

[16] C. Schwartz, Phys. Rev. 99, 1035 (1955).

[17] A.-M. Mårtensson-Pendrill, Phys. Rev. Lett. 74, 2184 (1995).

[18] H. H. Stroke, R. J. Blin-Stoyle, and V. Jaccarino, Phys. Rev. 123, 1326 (1961).

[19] S. Schmidt et al., Phys. Lett. B 779, 324 (2018).

[20] V. Mishin, V. Fedoseyev, H.-J. Kluge, V. Letokhov, H. Ravn, F. Scheerer, Y. Shirakabe, S. Sundell, and O. Tengblad, Nucl. Instrum. Methods B 73, 550 (1993).

[21] V. Fedosseev, K. Chrysalidis, T. D. Goodacre, B. Marsh, S. Rothe, C. Seiffert, and K. Wendt, J. Phys. G 44, 084006 (2017).

[22] B. A. Marsh, V. N. Fedosseev, and P. Kosuri, Hyperfine Interact. 171, 109 (2006)

[23] R. Catherall et al., J. Phys. G 44, 094002 (2017).

[24] R. N. Wolf et al., Int. J. Mass Spectrom. 349-350, 123 (2013).

[25] M. D. Seliverstov et al., Phys. Rev. C 89, 034323 (2014).

[26] J. G. Cubiss et al., Phys. Rev. C 97, 054327 (2018).

[27] E. A. Konovalova, M. G. Kozlov, Yu. A. Demidov, and A. E. Barzakh, Radiat. Appl. 2, 181 (2017).
[28] E. A. Konovalova, Yu. A. Demidov, M. G. Kozlov, and A. Barzakh, Atoms 6, 39 (2018).

[29] S. Büttgenbach, Hyperfine Interact. 20, 1 (1984).

[30] V. A. Dzuba, V. V. Flambaum, and M. G. Kozlov, Phys. Rev. A 54, 3948 (1996).

[31] M. G. Kozlov, S. G. Porsev, M. S. Safronova, and I. I. Tupitsyn, Comput. Phys. Commun. 195, 199 (2015).

[32] M. G. Kozlov, Int. J. Quant. Chem. 100, 336 (2004).

[33] M. S. Safronova, M. G. Kozlov, W. R. Johnson, and D. Jiang, Phys. Rev. A 80, 012516 (2009).

[34] V. A. Dzuba, V. V. Flambaum, M. G. Kozlov, and S. G. Porsev, Sov. Phys._JETP 87, 885 (1998).

[35] M. G. Kozlov, S. G. Porsev, and W. Johnson, Phys. Rev. A 64, 052107 (2001).

[36] T.-L Chen, I. Fan, H. C. Chen, C.-Y. Lin, S.-E. Chen, J.-T. Shy, and Y.-W. Liu, Phys. Rev. A 86, 052524 (2012).

[37] A. Lurio and A. G. Prodell, Phys. Rev. 101, 79 (1956).

[38] E. B. Baker and L. W. Burd, Rev. Sci. Instrum. 34, 238 (1963).

[39] E. Hagn, E. Zech, and G. Eska, Phys. Rev. C 24, 631 (1981).

[40] P. T. Callaghan, W. M. Lattimer, P. D. Johnston, and N. J. Stone, Hyperfine Interact. 2, 288 (1976).

[41] J. Eisinger and V. Jaccarino, Rev. Mod. Phys. 30, 528 (1958).

[42] E. Van Walle, D. Vandeplassche, J. Wouters, N. Severijns, and L. Vanneste, Phys. Rev. B 34, 2014 (1986).

[43] E. Van Walle, J. Wouters, D. Vandeplassche, N. Severijns, and L. Vanneste, Hyperfine Interact. 22, 507 (1985).

[44] E. Hagn and E. Zech, Nucl. Phys. A 399, 83 (1983).

[45] G. Eska, E. Hagn, T. Butz, P. Kienle, and E. Umlauf, Phys. Lett. B 36, 328 (1971).

[46] K. S. Krane and W. A. Steyert, Phys. Rev. C 9, 2063 (1974).

[47] W. Klinger, R. Böhm, W. Sandner, and W. Witthuhn, Nucl. Phys. A 350, 61 (1980).

[48] G. D. Alkhazov, A. E. Barzakh, I. Ya. Chubukov, V. P. Denisov, V. S. Ivanov, N. B. Buyanov, V. N. Fedoseyev, V. S. Letokhov, V. I. Mishin, and S. K. Sekatsky, Nucl. Phys. A 504, 549 (1989).

[49] A. E. Barzakh, I. Ya. Chubukov, D. V. Fedorov, V. N. Panteleev, M. D. Seliverstov, and Yu. M. Volkov, Phys. Rev. C 61, 034304 (2000).

[50] O. Häusser, P. Taras, W. Trautmann, D. Ward, T. K. Alexander, H. R. Andrews, B. Haas, and D. Horn, Phys. Rev. Lett. 42, 1451 (1979).

[51] K. H. Maier, J. A. Becker, J. B. Carlson, R. G. Lanier, L. G. Mann, G. L. Struble, T. Nail, R. K. Sheline, W. Stoff1, and L. Ussery, Phys. Rev. Lett. 48, 466 (1982). 
[52] A. Arima and H. Horie, Prog. Theor. Phys. 11, 509 (1954).

[53] G. Passler, J. Rikovska, E. Arnold, H.-J. Kluge, L. Monz, R. Neugart, H. Ravn, K. Wendt, and ISOLDE Collaboration, Nucl. Phys. A 580, 173 (1994).

[54] Y. W. Chan, W. B. Ewbank, W. A. Nierenberg, and H. A. Shugart, Phys. Rev. 137, B1129 (1965).
[55] W. B. Ewbank and H. A. Shugart, Phys. Rev. 135, A358 (1964). [56] G. Gould, Phys. Rev. 101, 1828 (1956).

[57] F. Le Blanc et al., Phys. Rev. Lett. 79, 2213 (1997).

[58] U. Krönert, St. Becker, G. Bollen, M. Gerber, Th. Hilberath, H.-J. Kluge, G. Passler, and ISOLDE Collaboration, Z. Physik A - Atom. Nucl. 331, 521 (1988). 